\title{
Diagnosing Paraproteinemic Keratopathy: A Case Report
}

\author{
Eugenie Mok ${ }^{a, b} \quad K a$ Wai Kam ${ }^{a, b}$ Anthony J. Aldave Alvin L. Young $^{a, b}$ \\ aDepartment of Ophthalmology \& Visual Sciences, The Chinese University of Hong Kong, \\ New Territories, Hong Kong, Hong Kong SAR; b Department of Ophthalmology \& Visual \\ Sciences, Prince of Wales Hospital, Hong Kong, Hong Kong SAR; 'Stein Eye Institute, \\ The University of California, Los Angeles, CA, USA
}

\section{Keywords}

Cornea $\cdot$ Corneal haze $\cdot$ Optical coherence tomography

\begin{abstract}
A 65-year-old man presented with bilateral, painless, progressive blurring of vision over 9 years. Slit-lamp examination revealed bilateral subepithelial corneal opacities in clusters located at the mid-periphery. Anterior segment optical coherence tomography, in vivo confocal microscopy (IVCM), serum protein electrophoresis, and molecular genetic testing were performed to evaluate the cause of corneal opacities. Anterior segment optical coherence tomography revealed a band-like, hyperreflective lesion in the Bowman layer and anterior stroma of both corneas. IVCM revealed hyperreflective deposits in the epithelium, anterior stroma, and endothelium. Serum protein electrophoresis identified the presence of paraproteins (immunoglobulin kappa), and molecular genetic testing revealed absence of mutations in the transforming growth factor beta-induced gene (TGFBI) and collagen type XVII alpha 1 gene (COL17A1). The ocular diagnosis of paraproteinemic keratopathy eventually led to a systemic diagnosis of monoclonal gammopathy of undetermined significance by our hematologist/oncologist. Paraproteinemic keratopathy is a rare differential diagnosis in patients with bilateral corneal opacities and therefore may be misdiagnosed as corneal dystrophy or neglected as scars. In patients with bilateral corneal opacities of unknown cause, serological examination, adjunct anterior segment imaging, and molecular genetic testing play a role in establishing the diagnosis.
\end{abstract}

\section{Introduction}

Paraproteinemia, otherwise known as monoclonal gammopathy, is the presence of a monoclonal protein in the blood. A monoclonal protein, or paraprotein, is an abnormal immunoglobulin or fragment of immunoglobulin produced in excess by a clonal proliferation of 
Mok et al.: Diagnosing Paraproteinemic Keratopathy

mature B-cells. This can be caused by a spectrum of diseases, both benign and malignant, including monoclonal gammopathy of undetermined significance (MGUS), multiple myeloma, chronic lymphocytic leukemia (CLL), non-Hodgkin lymphoma, Waldenström's macroglobulinemia, and amyloidosis.

MGUS is the most common form of paraproteinemia. It is a precursor to multiple myeloma or other plasma cell malignancies with a conversion rate of $1 \%$ per year. MGUS used to be considered a benign condition as the disease is defined by the absence of end organ damage. However, it is now recognized that MGUS can cause organ damage before reaching the predefined stage of lymphoma or multiple myeloma. This has given rise to terms such as monoclonal gammopathy of renal significance and monoclonal gammopathy of clinical significance. Although treatment is not recommended for MGUS under the current diagnostic schema, therapies targeting the underlying clonal protein appeared to achieve improvement in the neurologic or renal function of these patients [1]. Paraproteins are deposited in the kidneys during filtration across the glomeruli into the renal tubules. Similarly, paraproteins may deposit in the cornea (paraproteinemic keratopathy) via the tear film, diffusion from aqueous humor from the anterior chamber, or influx via the paralimbal vessels [2]. These multiple mechanisms of corneal deposition explain the variable presentation of paraproteinemic keratopathy.

We present a patient with bilateral corneal opacities mimicking a Bowman layer corneal dystrophy, with a final diagnosis of paraproteinemic keratopathy. We demonstrate that anterior segment imaging can locate subclinical corneal deposits and therefore plays an important role in the diagnosis. This case highlights the usefulness of anterior segment imaging and molecular genetic testing in differentiating between dystrophic and nondystrophic disorders with overlapping clinical features.

\section{Case Report}

A 65-year-old man of Chinese ethnicity presented to our clinic 9 years ago with chronic floaters. He did not complain of any blurring of vision at the time with a visual acuity of $20 / 20$ in both eyes. Slit-lamp biomicroscopy then showed multiple peripheral subepithelial corneal opacities in both eyes. The anterior chambers were quiet, and the lenses were clear. Fundal examination was unremarkable. There was no history of any corneal injury or family history of corneal disease. His underlying medical history included diabetes, hypertension, and hyperlipidemia.

His visual acuity remained stable until 6 years after initial presentation, when he started to complain of blurring of vision in the right eye, with a corresponding drop in visual acuity to $20 / 30$. He was then referred to our cornea clinic for consideration of cataract operation in the presence of the corneal opacities. He reported a gradual reduction in vision over the ensuing years and denied any symptoms of corneal epithelial erosions. Slit-lamp biomicroscopy revealed bilateral mid-peripheral subepithelial opacities in both eyes and also central mid to posterior stromal haze in the right eye (shown in Fig. 1). Fluorescein staining revealed mild punctate epithelial erosions and negative staining over some of the midperipheral subepithelial opacities. There was very mild cataract in both eyes, and hence cataract surgery was not recommended as the drop in vision was attributed to the increasing corneal opacities.

Anterior segment optical coherence tomography showed a hyperreflective band at the level of the Bowman layer and anterior stroma in both eyes, extending $160 \mu \mathrm{m}$ into the central stroma and $250 \mu \mathrm{m}$ into the paracentral stroma (shown in Fig. 2). The mid-peripheral, hyperreflective subepithelial opacities protruded toward the surface of the cornea and caused a

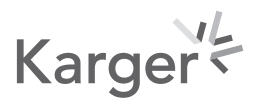


Fig. 1. a Slit-lamp photo of the right eye showing peripheral subepithelial opacities and central mid to posterior stromal haze. b Slit-lamp photo of the left eye showing multiple mid-peripheral granular subepithelial opacities.
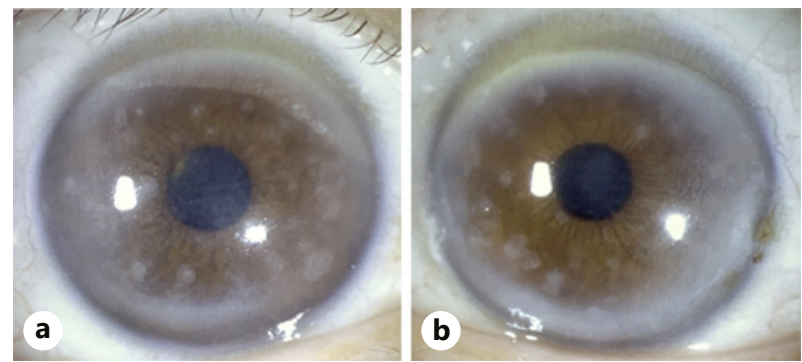

Fig. 2. AS-OCT images of the right eye showing uniformly hyperreflective bands in the Bowman layer and anterior stroma, occasional hyperreflectivity in the epithelium (arrow), corresponding thinning of the epithelium (arrowhead), and posterior stromal hyperreflective deposits (dotted arrows).

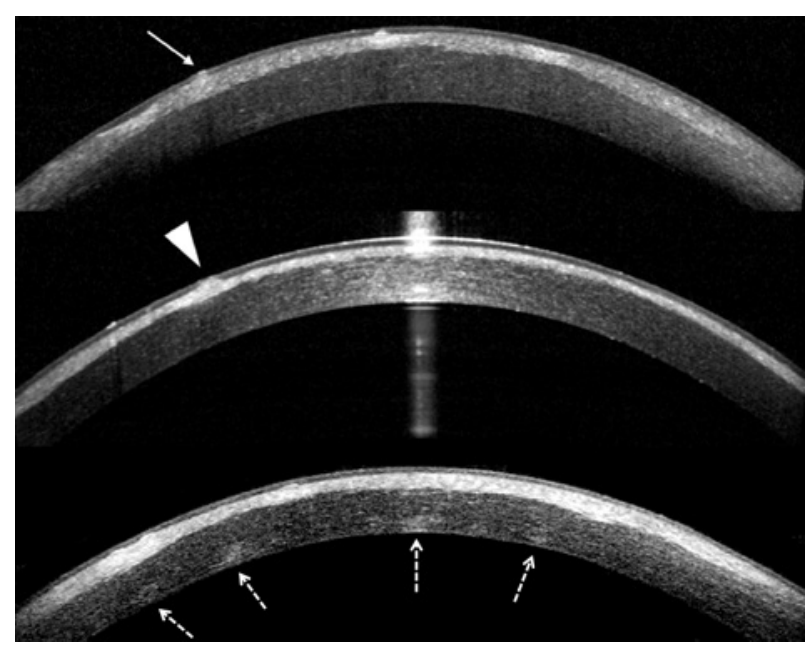

corresponding thinning of the overlying epithelium. There were hyperreflective deposits in the posterior stroma, more apparent in the right eye.

In vivo confocal microscopy (IVCM) of both corneas revealed hyperreflective deposits in the epithelium, anterior stroma, and endothelium (shown in Fig. 3). In the epithelium, there were small round discrete hyperreflective deposits. Mid-peripheral aggregates of these deposits were seen in areas corresponding to the opacities observed on slit-lamp examination. There was a generalized heterogeneous hyperreflectivity of the anterior stroma masking the appearance of the keratocytes. Hyperreflective deposits were not present in the mid and posterior stroma. The corneal nerves appeared normal in thickness but increased in tortuosity. There was no perineural deposit around the stromal nerves. Highly reflective granular deposits were present in the endothelium. The architecture of endothelial cells was preserved.

Given the presumed clinical diagnosis of a Bowman layer dystrophy or epithelial recurrent erosion corneal dystrophy (ERED), genetic testing was performed. Following the collection of saliva samples from the proband and his son, DNA was extracted from the saliva, and PCR amplification and Sanger sequencing were performed to screen the collagen type XVII alpha 1 gene (COL17A1) for the c.3156C>T mutations associated with ERED and all 17 exons of the transforming growth factor beta-induced gene (TGFBI). No pathogenic mutations were identified in TGFBI and the mutation associated with ERED was not identified in COL17A1, effectively excluding these corneal dystrophies as the cause of the corneal opacities.

Given the concern for a potential paraproteinemic keratopathy, the patient was referred to a hematologist/oncologist for further evaluation. Serologic evaluation revealed normal levels of total protein, serum IgG, IgA, and IgM. However, serum protein electrophoresis revealed a reduced level of albumin at $28 \mathrm{~g} / \mathrm{L}$ (normal range: $33-48 \mathrm{~g} / \mathrm{L}$ ) and the presence of 
Mok et al.: Diagnosing Paraproteinemic Keratopathy

Fig. 3. IVCM images of the right eye. a Epithelium $(29 \mu \mathrm{m})$ : aggregates of hyperreflective deposits. b Anterior stroma (88 $\mu \mathrm{m})$ : generalized heterogeneous hyperreflectivity masking the appearance of the keratocytes. c Midstroma $(330 \mu \mathrm{m})$ : keratocytes appear normal, and no hyperreflective deposits seen. d Endothelium $(470 \mu \mathrm{m})$ : multiple highly reflective granular deposits. IVCM, in vivo confocal microscopy.
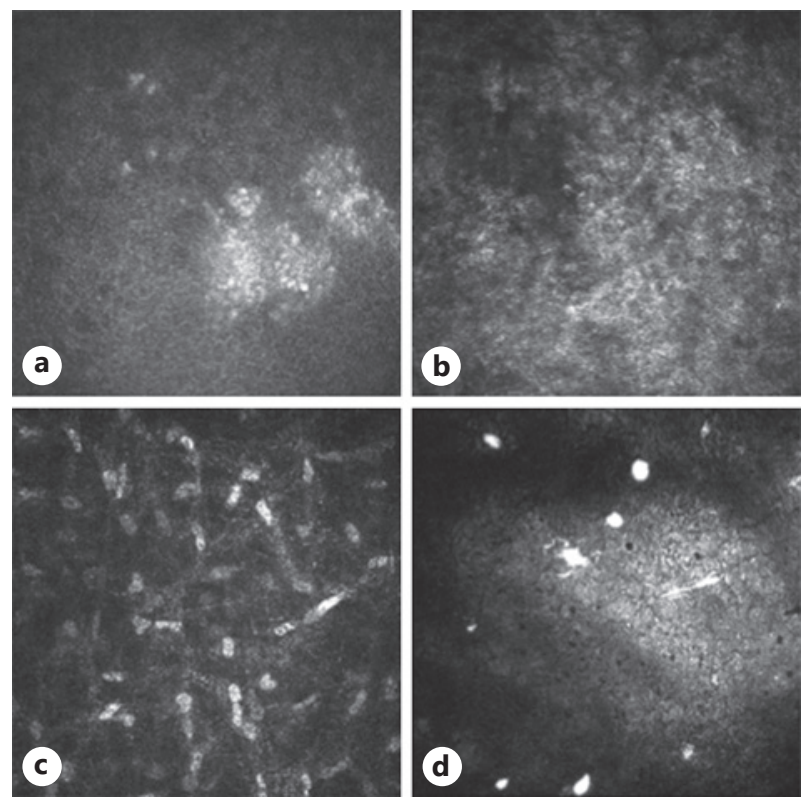

IgG kappa paraprotein at $7.6 \mathrm{~g} / \mathrm{L}$. Complete blood count, serum calcium level and renal function were normal. A bone marrow biopsy demonstrated $4 \%$ plasma cells and the presence of small atypical lymphoid aggregates of indeterminate nature. The skeletal survey showed no lytic lesions. These findings led to a diagnosis of MGUS.

\section{Discussion}

The clinical features of our patient resembled corneal dystrophy, in particular the Bowman layer or anterior stromal dystrophy within the TGFBI dystrophy spectrum. AS-OCT finding of hyperreflective material in the Bowman layer region and anterior stroma was also in keeping with typical presentation of corneal dystrophy.

Upon clinical examination, the differential diagnoses of corneal dystrophy include a myriad of metabolic disorders such as lecithin-cholesterol-acyltransferase deficiency, Fabry disease, tyrosine transaminase deficiency, and lysosomal storage diseases. Any cause of monoclonal gammopathy could lead to depositions of paraproteins and bilateral corneal opacities. Certain inherited skin diseases such as X-linked ichthyosis and keratosis follicularis spinolosa decalvans are associated with deposits in the corneas [3]. Molecular genetic testing plays an important role in diagnosing or excluding corneal dystrophy. At least 66 TGFBI mutations accountable for various corneal dystrophies have been identified [4]. Mutations in the TGFBI gene results in the gene product transforming growth factor $\beta$-induced protein, which accumulates in the cornea. The most common mutations are p.Arg124His, p.Arg124Cys, and p.Arg555Trp, which clinically correspond to Avellino corneal dystrophy, lattice corneal dystrophy, and granular corneal dystrophy, respectively [4].

The merit of anterior segment imaging in our patient lies in localizing the pathology and narrowing the differential diagnoses. Clinically, we could identify the subepithelial opacities readily on slit-lamp biomicroscopy. However, the stromal haze was faint and only seen in the right eye. The use of AS-OCT helped to clearly depict subepithelial opacities and posterior stromal hyperreflectivity in both eyes. IVCM also demonstrated endothelial deposits that were not apparent on slit-lamp biomicroscopy. The posterior involvement made the diagnosis 
of granular dystrophy less likely as granules only appear in the anterior two-thirds of stroma on AS-OCT [5].

In the literature, AS-OCT of paraproteinemic keratopathy has been described in 11 patients [6-13], with hematological diagnoses of MGUS [6-10], biclonal gammopathy of undetermined significance [7], smoldering multiple myeloma [11], multiple myeloma [9, 12], and CLL [13]. The features of AS-OCT in reported cases of paraproteinemic keratopathy are summarized in Table 1. All types of paraproteinemic keratopathy can have deposits in all layers of the cornea. There is no apparent association between the layer of deposits and the hematological diagnosis. The stroma is the most common site of paraprotein deposition in all types of paraproteinemia. Unlike previous case reports where anterior segment imaging only confirms clinically apparent corneal deposits, our case demonstrates that AS-OCT and IVCM can also locate subclinical corneal deposits that are not seen on slit-lamp examination.

Apart from the use of AS-OCT and IVCM, a Japanese group has demonstrated the use of corneal densitometry in analyzing paraproteinemic keratopathy. Ichii et al. [14] studied 30 patients with monoclonal gammopathy without visible corneal involvement on slit-lamp examination and found that they had significantly greater corneal densitometry values in the anterior and central cornea than the control group. This concludes that anterior segment imaging can be useful in picking up subclinical corneal depositions in monoclonal gammopathy.

Table 1. AS-OCT findings in reported cases of paraproteinemic keratopathy in the literature

\begin{tabular}{|c|c|c|c|c|c|}
\hline Age & Gender & Diagnosis & Paraprotein & Site of deposit & AS-OCT findings \\
\hline 43 & $\mathrm{M}$ & MGUS & IgG kappa & Whole stroma & $\begin{array}{l}\text { Homogenous hyperreflectivity of } \\
\text { stroma }\end{array}$ \\
\hline 43 & M & MGUS & IgG kappa & Whole stroma & $\begin{array}{l}\text { Diffuse haze throughout whole } \\
\text { stroma }\end{array}$ \\
\hline 77 & $\mathrm{~F}$ & MGUS & IgG lambda & $\begin{array}{l}\text { Posterior stroma, } \\
\text { Descemet } \\
\text { membrane }\end{array}$ & $\begin{array}{l}\text { Opacities in deep stromal layers and } \\
\text { at Descemet membrane }\end{array}$ \\
\hline 66 & M & MGUS & Карра & Anterior stroma & $\begin{array}{l}\text { Dense anterior stromal opacities with } \\
\text { significant distortion of anterior } \\
\text { corneal contour and variable } \\
\text { overlying epithelial thickness }\end{array}$ \\
\hline 36 & M & MGUS & IgG kappa & All layers & $\begin{array}{l}\text { Fine hyperreflective dot-shaped } \\
\text { deposits across all layers }\end{array}$ \\
\hline 59 & $\mathrm{~F}$ & $\begin{array}{l}\text { Biclonal } \\
\text { gammopathy of } \\
\text { undetermined } \\
\text { significance }\end{array}$ & $\begin{array}{l}\text { IgG lambda + } \\
\text { IgA lambda }\end{array}$ & $\begin{array}{l}\text { Descemet } \\
\text { membrane }\end{array}$ & Hyperreflectivity of posterior cornea \\
\hline 81 & $\mathrm{~F}$ & SMM & IgG light chain & All layers & $\begin{array}{l}\text { Small white crystalline deposits in all } \\
\text { layers }\end{array}$ \\
\hline 70 & M & MM & IgG lambda & Posterior stroma & $\begin{array}{l}\text { Highly reflective posterior stromal } \\
\text { deposit measuring } 500 \mu \mathrm{m} \text { with } \\
\text { significant distortion of posterior } \\
\text { corneal curvature }\end{array}$ \\
\hline 65 & $\mathrm{~F}$ & MM & Карра & Anterior stroma & $\begin{array}{l}\text { Very fine focal opacities with minimal } \\
\text { disruption of stromal architecture }\end{array}$ \\
\hline 85 & $\mathrm{~F}$ & MM & IgG kappa & Posterior stroma & $\begin{array}{l}\text { Multiple patchy gray-white deep } \\
\text { stromal opacities }\end{array}$ \\
\hline 53 & $\mathrm{~F}$ & CLL & IgG kappa & Anterior stroma & $\begin{array}{l}\text { Deposits with intervening clear } \\
\text { spaces }\end{array}$ \\
\hline
\end{tabular}

MGUS, monoclonal gammopathy of undetermined significance; SMM, smoldering multiple myeloma; MM, multiple myeloma; CLL, chronic lymphocytic leukemia. 
Mok et al.: Diagnosing Paraproteinemic Keratopathy

Paraproteinemic keratopathy is a rare differential diagnosis in patients with bilateral corneal opacities and therefore may be misdiagnosed as corneal dystrophy or neglected as scars. In patients with bilateral corneal opacities of unknown cause, it is important to consider a workup for paraproteinemia by serological examination and adjunct anterior segment imaging.

\section{Statement of Ethics}

This study was conducted ethically in accordance with the World Medical Association Declaration of Helsinki. Written informed consent was obtained from the patient for publication of this case report and accompanying images.

\section{Conflict of Interest Statement}

The authors have no conflicts of interest to declare.

\section{Funding Sources}

The authors have no funding sources to declare.

\section{Author Contributions}

K.W.K. followed up with the patient. A.J.A. facilitated the molecular genetic testing. E.M. and K.W.K. collected clinical data. E.M., K.W.K., A.J.A., and A.L.Y. contributed to the writing of the manuscript. All authors read and approved the final manuscript.

\section{References}

1 Lomas OC, Mouhieddine TH, Tahri S, Ghobrial IM. Monoclonal gammopathy of undetermined significance (MGUS)-not so asymptomatic after all. Cancers. 2020;12(6):1554.

2 Garibaldi DC, Gottsch J, de la Cruz Z, Haas M, Green WR. Immunotactoid keratopathy: a clinicopathologic case report and a review of reports of corneal involvement in systemic paraproteinemias. Surv Ophthalmol. 2005; 50(1):61-80.

3 Klintworth GK. Corneal dystrophies. Orphanet J Rare Dis. 2009;4, 1:7.4.

4 Lakshminarayanan R, Chaurasia SS, Anandalakshmi V, Chai SM, Murugan E, Vithana EN, et al. Clinical and genetic aspects of the TGFBI-associated corneal dystrophies. Ocul Surf. 2014;12(4):234-51.

5 Dalton K, Schneider S, Sorbara L, Jones L. Confocal microscopy and optical coherence tomography imaging of hereditary granular dystrophy. Cont Lens Anterior Eye. 2010;33(1):33-40.

6 Wasielica-Poslednik J, Gericke A, Desuki A, Schlötzer-Schrehardt U, Pfeiffer N, Lisch W. Recurrence of paraproteinemic keratopathy after penetrating keratoplasty and its assessment with confocal microscopy. Am J Ophthalmol Case Rep. 2018;11:87-91.

7 Lisch W, Wasielica-Poslednik J, Kivelä T, Schlötzer-Schrehardt U, Rohrbach JM, Sekundo W, et al. Erratum: the hematologic definition of monoclonal gammopathy of undetermined significance in relation to paraproteinemic keratopathy (an american ophthalmological society thesis). Trans Am Ophthalmol Soc. 2016;114: T7C1.

8 Karakus S, Gottsch JD, Caturegli P, Eghrari AO. Monoclonal gammopathy of "ocular" significance. Am J Ophthalmol Case Rep. 2019;15:100471.

9 Stiefel HC, Sandhu RK, Miller AK, Wilson DJ, Chamberlain WD. Characterization of corneal deposition keratopathy in the setting of blood cell dyscrasia and a minimally invasive technique to clear the cornea in a single case. Am J Ophthalmol Case Rep. 2019;13:83-8. 
10 Nobacht S, Kusters B, Breukink MB, Rongen GA, Cruysberg JRM. Recurrence of paraproteinemic crystalline keratopathy after corneal transplantation: a case of monoclonal gammopathy of ocular significance. Am J Ophthalmol Case Rep. 2020;19:100803.

11 Busch C, Koh S, Oie Y, Ichii M, Kanakura Y, Nishida K. In vivo confocal microscopy of multiple myeloma associated crystalline keratopathy. Am J Hematol. 2017;92(6):593-4.

12 Tainsh LT, Coady PA, Sinard JH, Neparidze N, Meskin SW, Adelman RA, et al. Asymmetric deep stromal keratopathy in a patient with multiple myeloma. Cornea. 2017;36(3):372-4.

13 Mannis TE, Mannis GN, Waterhouse EG, Aldave AJ, Rose-Nussbaumer J. Paraproteinemic keratopathy as the presenting sign of hematologic malignancy. Am J Hematol. 2016;91(9):961-2.

14 Ichii M, Koh S, Maeno S, Busch C, Oie Y, Maeda T, et al. Noninvasive assessment of corneal alterations associated with monoclonal gammopathy. Int J Hematol. 2019;110(4):500-5. 